\title{
Análise do uso de livros-texto digitais abertos no contexto da Educação Superior na América Latina
}

\author{
Ismar Frango Silveira \\ Universidade Presbiteriana Mackenzie - São Paulo, Brasil \\ ismar.silveira@mackenzie.br \\ Antonio Silva Sprock \\ Universidad Central de Venezuela - Caracas, Venezuela \\ asilva.sprock@gmail.com \\ Virginia Rodés Paragarino \\ Universidad de la República - Montevidéu, Uruguai \\ virginia.rodes@cse.edu.uy \\ Yván Jesús Túpac Valdivia \\ Universidad Católica San Pablo - Arequipa, Peru \\ ytupac@ucsp.pe
}

\begin{abstract}
Resumo. Entre as muitas barreiras para o acesso e permanência na Educação Superior nos países em desenvolvimento e subdesenvolvidos, como é o caso da totalidade dos países da América Latina, encontra-se o fator financeiro: ainda que não haja taxas de matrículas e mensalidades a serem pagas, há uma série de custos colaterais que nem sempre são considerados. Um destes custos é o dos materiais didáticos, em especial os livros-texto das disciplinas, de forma que iniciativas que possibilitem alternativas para os livros tradicionais devem ser consideradas. Neste sentido, o presente trabalho mostra os resultados da utilização de 25 livros-texto abertos gratuitos, criados de maneira colaborativa por autores de nove países Latino-americanas, disponibilizados de forma digital, no âmbito do Projeto LATIn. Um conjunto de 186 professores e 1.835 alunos espalhados em nove Universidades utilizou e avaliou as potencialidades e fragilidades deste modelo, e os resultados desta análise são apresentados neste artigo.
\end{abstract}

Palavras-chave: Livros de Textos Abertos, Escrita Colaborativa, Recursos Educacionais Abertos.

\section{Analysis of digital open textbooks use in the context of Latin America Higher Education}

Abstract. Amongst the many impediments for accessing and succeeding in Higher Education Institutions (HEI) in developing and underdeveloped countries, as in the totality of Latin American countries, are the financial factors: even though there are no tuition fees to be paid, there are many collateral costs that are somewhat overlooked. One of these costs is related to educational material, notably the cost of courses' textbooks, which leads to the need of considering alternatives to traditional textbooks. In this way, this paper shows the results of applying 25 open, freely available digital textbooks, created in a collaborative way by authors from 9 Latin American countries, in the context of LATIn Project. A group of 186 professors and 1,835 students spread through nine Universities used and evaluated the strengths and fragilities of this model, and the results of such analysis are here presented.

Keywords: Open textbooks, Collaborative Writing, Open Educational Resources 


\section{Introdução}

Nos países em desenvolvimento, e mais ainda em países subdesenvolvidos, o custo dos livros em ensino de graduação é muitas vezes proibitivo para a maioria dos estudantes, fazendo com que esses livros sejam inacessíveis a eles, motivando-os, muitas vezes, a recorrer a cópias ilegais, seja em papel ou digitais. Um estudo feito na maior universidade do Brasil (USP - Universidade de São Paulo) mostra que a compra de livros usados nos cursos do campus USP-Leste é incompatível com a média salarial das famílias dos alunos (Craveiro et al., 2008). O estudo citado mostra igualmente a disponibilidade dos livros - em oposição à reprografia, que no Brasil é ilegal em se tratando da obra completa e sem o recolhimento dos direitos autorais, há obras presentes nos Projetos Pedagógicos dos Cursos que não estão disponíveis para compra, estando esgotadas nas livrarias.

Este problema tem sido tradicionalmente resolvido através de bibliotecas universitárias, que emprestam cópias dos livros didáticos gratuitamente para os alunos. No entanto, as bibliotecas universitárias não têm orçamento suficiente para atender a demanda de todo os alunos - não é raro encontrar uma taxa de 50 alunos por livro em cursos de alta demanda. Isto impacta em especial os alunos de baixa renda, visto que os custos de aquisição de livros são muito altos para o poder de compra da maioria das famílias. Desta forma, proliferam soluções alternativas, porém ilegais em sua maioria: fotocópias de livros em papel e downloads de livros digitais disponibilizados à revelia dos autores e editoras encontram-se entre as soluções - ilegais, reitere-se - encontradas pelos alunos, que por sua vez teriam como única alternativa legal a utilização exclusiva de suas próprias notas de aula como fonte de consulta, além dos materiais cedidos pelos professores - estes, também, passíveis de uma análise de conformidade com os marcos legais de cada país. Esse problema cria uma diferença significativa entre os estudantes que podem ter uma cópia do livro didático e aqueles que não podem.

Uma das razões para o alto custo dos livros didáticos é que, em muitos países subdesenvolvidos e em desenvolvimento, são produzidos no exterior. No contexto da América Latina, o Brasil possui a maior produção editorial e é responsável por mais da metade dos livros editados globais no continente, seguido pelo México, Argentina, Colômbia, Chile e Venezuela (Lindoso, 2004). No entanto, a produção total de livros na região é efetivamente menor do que $10 \%$ de todo o mundo.

Além disso, de acordo com vários estudos, apenas uma pequena parte (aproximadamente 10-20\%) dos livros utilizados nas instituições de ensino superior da América Latina foram criadas por autores latino-americanos. Assim, a raiz do problema não está relacionada com a falta de capacidade de produção, mas com a dificuldade que os professores locais e autores têm de publicar e distribuir seus livros.

Além disso, Craveiro et al. (2008) também mostraram que os autores estrangeiros (traduzidos ou adotados sem tradução) têm uma forte presença na bibliografia dos cursos, chegando a representar $64,5 \%$ dos livros presentes na bibliografia básica de um dos cursos analisados no estudo. Quando importados diretamente, mesmo sem impostos (o que ocorre em alguns países), esses livros seguem sendo mais caros, devido aos custos de frete; quando (muitas vezes mal) traduzidos, estes livros contribuem para desmotivar os autores locais a produzirem suas próprias obras.

O problema com a origem estrangeira dos livros didáticos têm várias consequências adicionais além de seu custo: muitos dos livros didáticos não se mostram adequados ao contexto dos sistemas de ensino superior dos países subdesenvolvidos ou em 
desenvolvimento. Ainda, as versões mais recentes do livro demoram a estar disponíveis, quando o são, na língua nativa dos professores e os alunos, o que cria um problema de fundo cultural: faz surgir a percepção prejudicial entre os alunos que o conhecimento sempre vem de fora da região.

Uma alternativa para melhorar o acesso aos livros didáticos de nível universitário surgiu no campo das tecnologias educativas, desde o conceito de Recursos Educacionais Abertos (REA), que se referem aos materiais didáticos, geralmente digitais, que podem ser livremente copiados, modificados, partilhados, impressos e distribuídos, o que contribuiu para a criação do conceito de livros-texto abertos (UNESCO, 2002). Este tipo de material didático oferece potenciais benefícios para a Educação, uma vez que permite reduzir o tempo e os custos associados ao processo de concepção e produção de livros didáticos, através de sua adaptação, reutilização e remixagem.

Neste sentido, nasceu o Projeto LATIn (Latin American open Textbooks Initiative, www.latinproject.org), cujo objetivo inicial foi "estabelecer uma iniciativa para a criação colaborativa de livros de texto abertos para a educação superior na América Latina" (Ochôa et al, 2011).

A iniciativa se estabeleceu entre 2011 e 2014 no marco do (hoje extinto) Projeto trienal Alfa III (DCIALA/19.09.01/11/21526/279-155/ALFA III (2011)-52), com financiamento da União Europeia, envolvendo nove países da América Latina, com o apoio de três universidades europeias (advindas da Espanha, França e Bélgica). O desenvolvimento do Projeto, o que envolveu a análise do estado-da-arte, definição de metodologias de escrita colaborativa e demais marcos teóricos e legais, desenvolvimento de plataformas computacionais de suporte, estabelecimento de estratégias de adoção e difusão, bem como a aplicação do plano piloto, ficou a cargo de uma universidade por país. A Figura 1 mostra a área de abrangência inicial do Projeto LATIn (países em cinza):

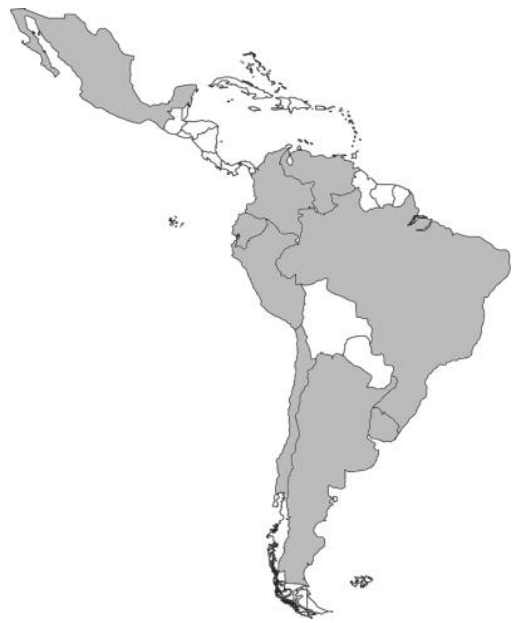

Figura 1. Área de abrangência inicial do Projeto LATIn

Em seu plano-piloto, LATIn previa a criação de livros digitais de maneira colaborativa, disponíveis para download em formato eletrônico, podendo ser impressos sem custos relacionados a direitos de licenciamento. Objetivou-se que os livros fossem disponibilizados em formatos que permitissem sua reutilização, remixagem, redistribuição, revisão e retenção da licença aberta, de acordo com os princípios 5R (retain, reuse, revise, remix, redistribute) dos REA (Wiley, 2014). Isto permite a criação de livros personalizados: cada professor pode selecionar seções apropriadas para o curso, adaptar ou criar novos elementos para o livro livremente, gerando sua própria 
versão. Espera-se que esta iniciativa encoraje os professores de diferentes instituições na região para trabalhar colaborativamente na criação de capítulos e livros didáticos adaptados às necessidades específicas de seus cursos e seus contextos sócio-culturais. Além disso, os alunos terão acesso gratuito a estes livros, o que representa uma alternativa que contribui para reduzir o impacto do custo dos livros didáticos.

O Projeto foi planejado ao redor de três eixos principais (Deco et al., 2013) (Silveira et al., 2013a), pautado pelas questões pedagógicas, tecnológicas e políticas necessárias para fazer este tipo de iniciativa ser bem sucedido no contexto da América Latina. Os eixos de trabalho estão intimamente relacionados, de maneira que as decisões tomadas em cada um deles impactavam nos demais, estabelecendo um ecossistema digital (Silveira et al, 2013b).

No primeiro eixo, teórico-metodológico, projetou-se uma metodologia para a criação colaborativa de livros didáticos abertos, com ênfase nos aspectos legais e nos processos colaborativos (Casali et al. , 2012). O segundo eixo, tecnológico, envolveu o projeto e implementação de plataformas tecnológicas para apoiar a criação colaborativa, adaptação, combinação e reutilização de livros abertos (Ochôa et al., 2013). O terceiro e último eixo tratava de estratégias: definiu-se um conjunto de estratégias para a implementação e adoção dessa iniciativa nas instituições de ensino superior na região (Rodés et al., 2013).

No âmbito do eixo 2, foram desenvolvidas duas plataformas computacionais: uma plataforma social para formação dos grupos de escrita e uma plataforma de escrita colaborativa, ambas implementadas sob a filosofia de mashups e disponibilizadas através de licenças abertas. Estas plataformas permitiram o surgimento de 94 comunidades de escrita, das quais surgiram 47 propostas de livros-texto abertos. Destas propostas, selecionaram-se 25 para serem desenvolvidos colaborativamente por 144 professores-autores- detalhamentos sobre este processo podem ser encontrados em Silva Sprock (2014). Esses livros, uma vez elaborados, foram utilizados e avaliados por 186 professores e 1.835 alunos. Os resultados desta avaliação encontram-se no item a seguir.

\section{Aplicação e avaliação dos livros por professores}

De forma a conhecer a opinião dos potenciais usuários sobre os livros-texto desenvolvidos, dois questionários foram criados on-line, dirigidos aos professores que efetivamente utilizaram os livros em suas disciplinas entre 2013 e 2014. A avaliação atingiu 186 professores de diferentes áreas, em quantidades diferentes por país, como pode ser analisado na Figura 2 a seguir.

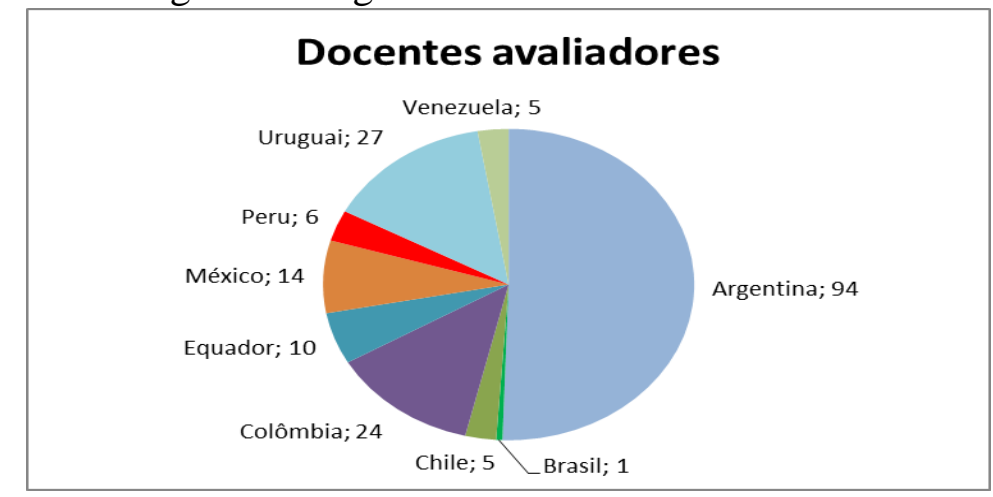

Figura 2. Profes sores que utilizaram e avaliaram os livros -texto abertos 
Deve-se ressaltar que a baixa participação de professores brasileiros se deu basicamente pelo fato de que quase todos os livros estavam unicamente escritos em espanhol, nesta fase - atualmente vêm sendo traduzidos para o português. Um aspecto que deve ser levado em consideração é que uma parcela razoável dos professores consultados também atuou na escrita do livro. Os elementos avaliados no instrumento foram os seguintes, através de perguntas com respostas abertas:

1. O livro se mostrou adequado para o seu curso?

2. Qual a percentagem dos conteúdos do curso que você estima que tenha sido coberta neste livro?

3. Como foi a recepção do livro pelos alunos?

4. Você identificou problemas usando o livro? Quais?

5. Você está disposto em participar na melhoria este livro, adicionando conteúdo, exemplos, ajudando a adaptá-lo, traduzi-lo, etc.?

6. Que recomendações você faria para aumentar o uso de livros abertos no contexto universitário?

7. Outros comentários.

Uma análise geral das respostas dos professores mostrou uma aceitação bastante forte e uma postura positiva a respeito dos livros - entretanto, este posicionamento pode ter sido influenciado pela forte presença de professores-autores na amostra trabalhada, que já estavam envolvidos no processo. Dados importantes surgiram da pergunta 2, a respeito da percepção de cobertura dos livros em relação ao conteúdo da disciplina. A Figura 3 exibe os dados obtidos:

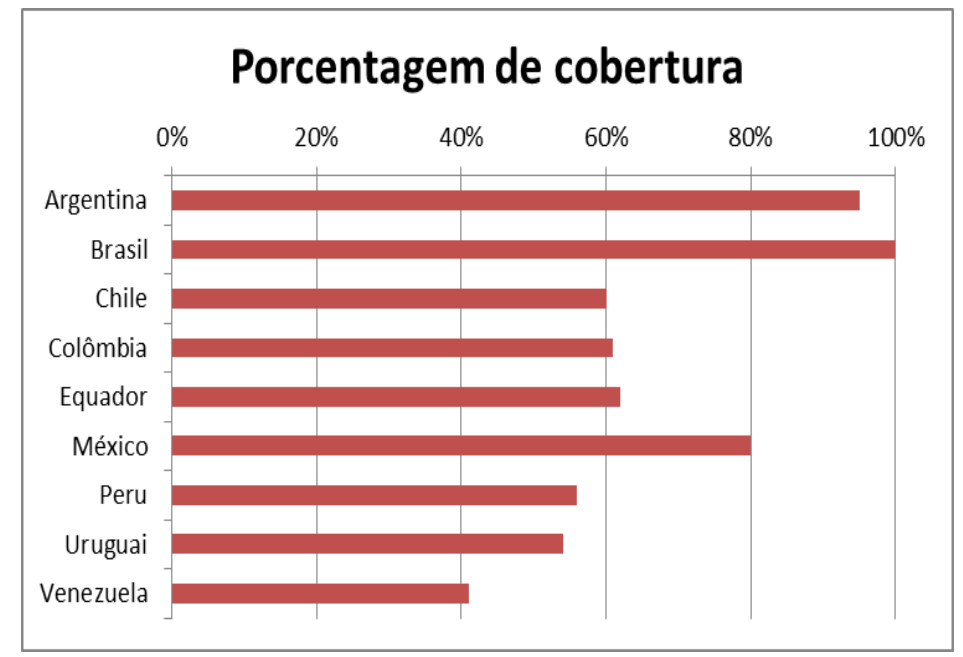

Figura 3. Avaliação da cobertura conceitual dos livros por parte dos professores.

Frente ao gráfico da Figura 3, algumas inferências podem ser obtidas: Por exemplo, o resultado de $100 \%$ obtido no Brasil deve-se ao fato de somente um professor ter participado da avaliação, o que não é estatisticamente significativo. Da mesma maneira, o baixo resultado obtido na Venezuela pode ter sido influenciado pelo - também relativamente baixo - número de professores avaliadores, que se limitou a um conjunto de 5 professores. O país com mais avaliadores, a Argentina (94 professores) apresentou a melhor avaliação de cobertura (apenas atrás do Brasil), na média de 95\%. Uma análise global dos dados resulta em uma média avaliação de cobertura de $78 \%$, o que é bastante significativo. 


\section{Uso e avaliação dos livros pelos estudantes}

A Tabela 1, a seguir, exibe o número de alunos que avaliaram os livros-texto.

Tabela 1. Número de alunos que utilizaram e avaliaram os 25 livros -texto abertos

\begin{tabular}{|c|c|c|c|}
\hline Livro & $\begin{array}{l}\text { Países de } \\
\text { aplicação }\end{array}$ & $\mathbf{E} / \mathbf{U}$ & TE \\
\hline Álgebra Lineal para Ingeniería & Chile & 73 & 73 \\
\hline Anatomía de las Arterias de la Cabeza & México & 28 & 28 \\
\hline Aplicaciones y teoría de ingeniería de microondas & Peru & 10 & 10 \\
\hline Aprendizaje Colaborativo apoyado por Computador & $\begin{array}{l}\text { Venezuela } \\
\text { Colômbia }\end{array}$ & $\begin{array}{c}21 \\
9\end{array}$ & 30 \\
\hline Cálculo Diferencial e Integral & Argentina & 24 & 24 \\
\hline Comercio Internacional & Chile & 37 & 37 \\
\hline $\begin{array}{l}\text { Diseño e Implementación de Bases de Datos desde una Perspectiva } \\
\text { Práctica }\end{array}$ & $\begin{array}{l}\text { Equador } \\
\text { Colômbia } \\
\text { México } \\
\end{array}$ & $\begin{array}{c}5 \\
1 \\
17 \\
\end{array}$ & 23 \\
\hline $\begin{array}{l}\text { Economía Internacional. Claves teórico prácticas sobre la inserción de } \\
\text { Latinoamérica en el mundo }\end{array}$ & $\begin{array}{l}\text { Argentina } \\
\text { Colômbia }\end{array}$ & $\begin{array}{c}28 \\
1\end{array}$ & 29 \\
\hline $\begin{array}{l}\text { Educación Alimentaria y Nutricional en el marco de la Educación para } \\
\text { la Salud }\end{array}$ & $\begin{array}{l}\text { Uruguai } \\
\text { Venezuela }\end{array}$ & $\begin{array}{c}23 \\
376 \\
\end{array}$ & 399 \\
\hline $\begin{array}{l}\text { Elementos esenciales para programación: Algoritmos y Estructuras de } \\
\text { Datos }\end{array}$ & $\begin{array}{l}\text { Equador } \\
\text { Uruguai } \\
\text { Argentina }\end{array}$ & $\begin{array}{l}63 \\
63 \\
59 \\
\end{array}$ & 185 \\
\hline Estructura de Datos & $\begin{array}{l}\text { Equador } \\
\text { Colômbia }\end{array}$ & $\begin{array}{l}77 \\
11\end{array}$ & 88 \\
\hline Estudios del lenguaje: Niveles de representación lingüística & $\begin{array}{l}\text { Uruguai } \\
\text { Argentina }\end{array}$ & $\begin{array}{c}5 \\
93\end{array}$ & 98 \\
\hline Gestión de Proyectos de Software & México & 27 & 27 \\
\hline Inteligencia Artificial & $\begin{array}{l}\text { Chile } \\
\text { Peru } \\
\text { Venezuela }\end{array}$ & $\begin{array}{l}20 \\
18 \\
34 \\
\end{array}$ & 72 \\
\hline Introducción al Estudio de Fuentes Renovables de Energía & Equador & 37 & 37 \\
\hline $\begin{array}{l}\text { Objetos de Aprendizaje de Contenidos Abiertos Accesibles: del Diseño } \\
\text { a la Reutilización }\end{array}$ & $\begin{array}{l}\text { Chile } \\
\text { Venezuela } \\
\text { Colômbia }\end{array}$ & $\begin{array}{c}4 \\
20 \\
1\end{array}$ & 25 \\
\hline Planes de Negocios para Emprendedores & $\begin{array}{l}\text { Peru } \\
\text { Uruguai }\end{array}$ & $\begin{array}{l}11 \\
35\end{array}$ & 46 \\
\hline Política Monetaria Fiscal & Chile & 11 & 11 \\
\hline Políticas Públicas, Género y Derechos Humanos en América Latina & Argentina & 50 & 50 \\
\hline Promoción de la Salud. Una pers pectiva latinoamericana & $\begin{array}{l}\text { Argentina } \\
\text { México }\end{array}$ & $\begin{array}{c}240 \\
36\end{array}$ & 276 \\
\hline Sentido y Características de las Ciudades Modernas & $\begin{array}{l}\text { Brasil } \\
\text { Uruguai } \\
\text { Argentina }\end{array}$ & $\begin{array}{l}18 \\
85 \\
12 \\
\end{array}$ & 115 \\
\hline Sistemas Operativos & $\begin{array}{l}\text { Argentina } \\
\text { Colômbia } \\
\text { México }\end{array}$ & $\begin{array}{c}8 \\
1 \\
10 \\
\end{array}$ & 19 \\
\hline Temas de Diseño en Interacción Humano Computadora & $\begin{array}{l}\text { Colômbia } \\
\text { México }\end{array}$ & $\begin{array}{l}25 \\
50\end{array}$ & 75 \\
\hline Temas de Teoría de la Computación & $\begin{array}{l}\text { Uruguai } \\
\text { Argentina }\end{array}$ & $\begin{array}{l}10 \\
20\end{array}$ & 30 \\
\hline Tópicos avanzados de Bases de datos & $\begin{array}{l}\text { Equador } \\
\text { Argentina } \\
\text { México }\end{array}$ & $\begin{array}{c}14 \\
5 \\
9 \\
\end{array}$ & 28 \\
\hline
\end{tabular}


$\mathrm{Na}$ tabela 1, E/U representa o número de estudantes por país, enquanto TE representa o número total de estudantes. Os aspectos avaliados no instrumento aplicado aos 1.835 estudantes matriculados em disciplinas que utilizaram os livros-texto do Projeto LATIn foram organizados em 11 questões, mostradas abaixo na Tabela 2:

Tabela 2: Aspectos avaliados pelos estudantes

Aspectos avaliados

\begin{tabular}{l|l}
\hline A & Este livro foi muito útil para estudaro assunto \\
B & Este livro usa uma linguagem de acordo com o meu contexto cultural \\
$\mathbf{C}$ & Eu gosto da organização dos conteúdos do livro \\
D & Eu gosto dos exercícios e/ou atividades do livro \\
$\mathbf{E}$ & Este livro me motiva a estudar \\
$\mathbf{F}$ & Este livro me facilitou a compreender a matéria \\
$\mathbf{G}$ & Eu prefiro este livro do que os livros tradicionais para esta matéria \\
$\mathbf{H}$ & Recomendaria este livro para outros estudantes \\
I & Este livro me ajudou a reduzir as despesas commaterial de estudo \\
J & A qualidade do conteúdo do livro é excelente \\
$\mathbf{K}$ & Este livro poderia substituiralguns dos livros tradicionais do curso
\end{tabular}

As respostas foram dadas de acordo com uma Escala de Likert de cinco pontos. Dentre todos os livros avaliados, o livro que obteve resultados estatisticamente mais significativos foi a obra "Educación Alimentaria y Nutricional en el marco de la Educación para la Salud", tendo sido usado por 399 estudantes na Venezuela e Uruguai. A Figura 4, a seguir, mostra, à guisa de exemplo, os dados relativos à avaliação deste livro pelos estudantes.

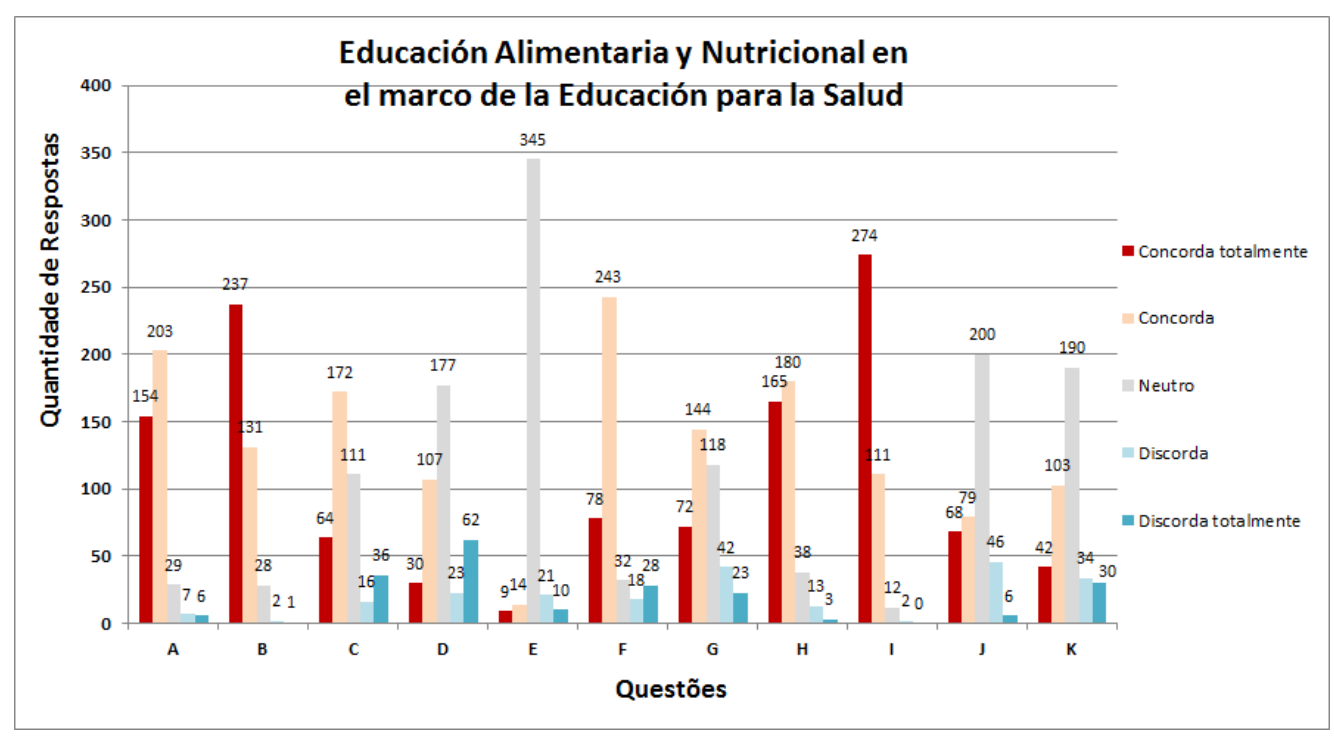

Figura 4. Resultados da avaliação de um livro na área da Saúde

Chama a atenção a resposta neutra à questão E ("Este livro me motiva a estudar") dada por $87 \%$ (ou 345) estudantes. Também prevalece a mesma resposta neutra para as perguntas J e K ("A qualidade do conteúdo do livro é excelente" e "Este livro poderia substituir alguns dos livros tradicionais de curso"), correspondendo a $50 \%$ e $47 \%$, respectivamente. Por outro lado, 96\% (385 estudantes) concordam ou concordam fortemente que o livro ajuda a reduzir os custos dos estudos (pergunta I). 
Os resultados individuais da avaliação de cada livro não serão mostrados aqui por questões de espaço. Entretanto, é importante ressaltar que as idiossincrasias das áreas de conhecimento de cada livro parecem interferir nos resultados. Por exemplo, há um número significativo de respostas neutras à questão I para os livros da área de Informática, relativa ao fato dos livros, por serem abertos e gratuitos, ajudarem a reduzir os custos com materiais. Enquanto o livro analisado na Figura 4, da área de Saúde, apresentou somente $3 \%$ de respostas neutras (e $0,5 \%$ de respostas negativas) a esta questão, o livro "Elementos esenciales para programación: Algoritmos y Estructuras de Datos" apresenta 19\% de respostas neutras. Da mesma forma, o livro "Estructuras de Datos" tem 16\% de respostas neutras nesse quesito. Estes dados podem talvez ser explicados pelo perfil dos estudantes dos cursos de Computação e Informática, que geralmente buscam fontes de informação na Internet, seja de maneira legal ou não, mas geralmente sem qualquer custo para eles

Os resultados consolidados da avaliação de todos os 25 livros do Projeto LATIn podem ser vistos na Figura 5 a seguir:

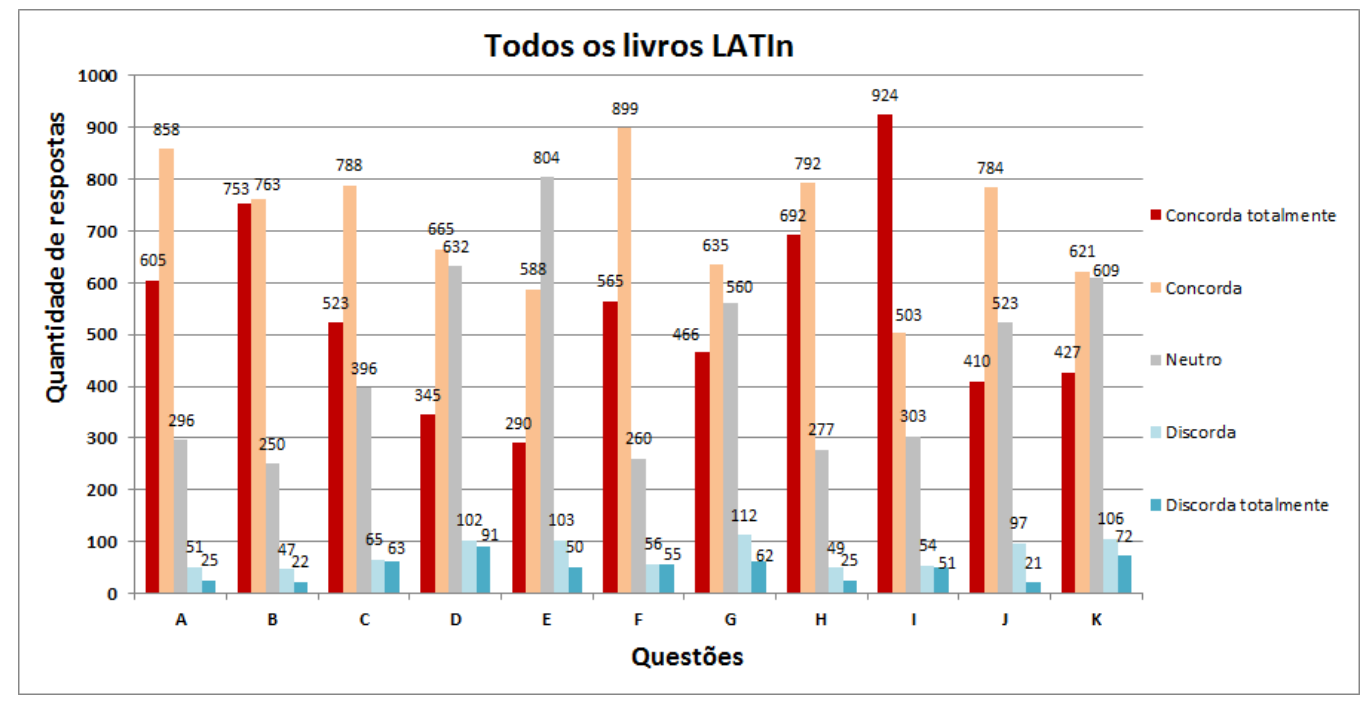

Figura 5. Resultados consolidados para todos os livros LATin

A figura 5 apresenta dados importantes para a análise da aceitação e impacto dos livros para os estudantes. As respostas às perguntas A ("Este livro foi muito útil para estudar o assunto"), H ("Recomendaria este livro para outros estudantes") e I ("Este livro me ajudou a reduzir as despesas com material de estudo") apresentaram o maior índice de respostas positivas $(80 \%, 81 \%$ e $78 \%$, entre "concordo" e "concordo totalmente"). Embora os resultados positivos para a pergunta I fossem plenamente esperados (mais de $50 \%$ das respostas "concordo totalmente", a maior taxa entre todas as perguntas), por ser o alto custo dos livros tradicionais um dos motivadores Projeto LATIn, percebe-se nas respostas dos estudantes um alto grau de aceitação dos livros, a ponto de fazer com os alunos concordassem em recomendar os livros-texto abertos aos seus colegas (pergunta $\mathrm{H}$ ), confirmando a utilidade dos livros para esse grupo de alunos investigados (pergunta A).

Claramente, também são dignas de nota as respostas negativas $(10 \%$ entre "discordo" e "discordo totalmente") para a questão D: "Eu gosto dos exercícios e/ou atividades do libro". Este fator deve ser levado em consideração em novas versão desses livros e livros novos que venham a ser criados sob a iniciativa LATIn. E outro aspecto que merece atenção é o alto grau de indiferença sobre a questão E: "Este livro me motiva 
estudar" (44\% dos estudantes mostraram-se "neutros" quanto a este aspecto). Isso leva à necessidade de investigar os motivos e as circunstâncias que envolvem aspectos motivacionais no processo de aprendizagem, já que livros, mesmo que abertos e gratuitos, não aparentam ser um fator de motivação e inovação didática para muitos dos estudantes. Ações futuras que envolvam outros recursos de mídia educativa aberta (vídeos, simulações e jogos abertos, por exemplo) e abordagens inovadoras de desenho instrucional podem ser capazes de proporcionar impactos mais significativos sobre $\mathrm{o}$ aspecto motivacional dos estudantes.

\section{Conclusões}

Uma vez avaliados os 25 livros desenvolvidos no plano piloto do Projeto LATIn por meio de questionários aplicados a professores e estudantes de 9 universidades latinoamericanas, foi possível validar o potencial de impacto da iniciativa. O Projeto LATIn, proposto inicialmente para desenvolver soluções que atacassem problema do alto custo dos livros didáticos para o Ensino Superior na América Latina, terminou por gerar um conjunto de diretrizes, metodologias, estratégias e plataformas computacionais que permitem que seus princípios possam ser adequados às necessidades específicas de outros países, regiões línguas e culturas.

Como resultado da pesquisa feita com os professores, obteve-se uma avaliação bastante positiva da iniciativa, destacando a formação efetiva de grupos de professores-autores por meio das plataformas tecnológicas desenvolvidas pelo Projeto. Por outro lado, uma série de melhorias apontadas pelos professores nestas mesmas plataformas devem ser levadas em consideração em versões futuras das mesmas.

No que diz respeito aos estudantes, é importante destacar a contribuição do Projeto LATIn no sentido de se ter alternativas de redução de custos com materiais de estudo, além de uma percepção positiva quanto à adaptação dos livros-texto aos diferentes contextos culturais dos alunos, sendo estes parte dos principais objetivos do Projeto.

Entre as ações empreendidas ao final do Projeto LATIn, destaca-se a criação da Associação LATIn - Editora Aberta, organização social sem fim de lucro, com sede em Montevidéu, cuja acordo de criação foi assinado pela maioria dos participantes do Projeto LATIn, além de outros pesquisadores interessados na proposta, no mês de outubro de 2014.

\section{Referências}

Craveiro,G.; Machado, J.; Ortellado, P. (2008) O mercado de livros técnicos e científicos no Brasil: subsídio público e acesso ao conhecimento. Disponível na Internet em http://www.gpopai. usp.br/relatoriolivros.pdf. Acesso em 20 de abril de 2015.

Casali, A., Silva Sprock, A., Alves, C., Deco, C., Silveira, I. F., Muñoz-Arteaga, J., Gomes dos Santos, J., Broisin, J., Morales, R., C Hernández, Y., Ochoa, X. (2012). Collaborative Methodologies for Writing Open Educational Textbooks: a State-of art Review. Vol. 1(1) In: Anais dos Workshops do Congresso Brasileiro de Informática na Educação, 2012. Rio de Janeiro, Brasil.

Deco, C., Casali, A., Bender, C., Kantor, R., Motz, R. (2013). Creación de libros abiertos en América latina: metodología, plataforma y estrategias. Actas de las XV Workshop de Investigadores en Ciencias de la Computación, 2013. Paraná, Argentina. 
Lindoso, F. (2004) O Brasil pode ser um país de leitores? Política para cultura/ politica para o livro. São Paulo: Summus Editorial.

Ochôa, X.; Silva Sprock, A.; Silveira, I. F. (2011). Collaborative Open Textbooks for Latin America - The LATIn Project. In: Proceedings of Information Society Conference (i-Society 2011), Londres, pp.398-403.

Ochoa, X. ; Casali, A. ; Deco, C. ; Gerling, V. ; Silveira, I. F. ; Fager, J. ; Carillo, G. ; Parra, G. ; Arteaga, J. M. ; Maturana, J. ; Araya, E. ; Motz, R. (2013). Analysis of Existing Technological Platforms for the Collaborative Production of Open Textbooks. In: EdMEDIA'2013 - World Conference on Educational Multimedia, Hypermedia and Telecommunications 2013, 2013, Victoria, Canadá. In: EdMEDIA'2013 - Proceedings of World Conference on Educational Multimedia, Hypermedia and Telecommunications 2013. Chesapeake, VA, Canadá: AACE, 2013. p. 1106-1115

Rodés, V., Rodríguez, C., Maturana, J., Hernández, Y., Cuadros Vargas, E., Podetti, M. (2013). Strategies for Implementing the Adoption of Open Textbooks Initiatives: State of the Art Review. In: International Journal of e-Education, e-Business, eManagement and e-Learning (IJEEEE 2013). Vol.3(1): 51-56 ISSN: 2010-3654.

Silveira, I. F., Mustaro, P., Silva, L., Nizam, O., Knihs, E., Rodés, V, Silva Sprock, A., Ochoa, X. (2013). O desafio do acesso e permanência no Ensino Superior frente ao custo dos livros didáticos: proposta de uma arquitetura de criação e disseminação de livros digitais colaborativos abertos. In: Proceedings do XXXIII Congresso da Sociedade Brasileira de Computação 2013 (CSBC2013). II Workshop de Desafios da Computação Aplicada à Educação (DEsafIE!2013), Julio 2013. Maceió. Brasil.

Silveira, I. F., Ochoa, X., Cuadros-Vargas, A., Casas, A.P., Casali, A., Ortega, A., Silva Sprock, A., Alves, C., Collazos, C., Deco, C., Cuadros-Vargas, E., Knihs, E., Parra, G., Muñoz-Arteaga, J., dos Santos, J.G., Broisin, J., Omar, N., Motz, R., Rodés, V., Hernández, Y. (2013). Digital Ecosystem for the Collaborative Production of Open Textbooks: The LATIn Methodology. In: Journal of Information Technology Education: Research. Vol. 12, pp. 225-249.

Silva Sprock, A. (2014). Resultados del Plan Piloto de LATIn. In: Semana de los Libros Abiertos de RedCLARA. Disponível na Internet em https://eventos.redclara.net/ indico/event/454/page/4. Acesso em 3 de julho de 2015.

UNESCO. (2002). Open Educational Resources - Open Content for Higher Education - Final Forum Report. Disponível na Internet em http://portal.unesco.org/ci/en/files/21713/11438000259OER__ Forum_Final_Report.pdf/. Acesso em 20 de abril de 2015.

Wiley, D. (2014) The Access Compromise and the 5th R. Disponível na Internet em http://opencontent.org/blog/archives/3221. Acesso em 20 de abril de 2015.

\section{Agradecimentos}

Os autores deste artigo agradecem o apoio da União Europeia por meio do Projeto Alfa III: DCI-ALA/19.09.01/11/21526/279-155/ALFA III (2011)-52. 